\title{
Características da População com Sepse em Unidade de Terapia Intensiva de Hospital Terciário e Privado da Cidade do Recife*
}

\author{
Characteristics of Septic Patients in an Intensive Care Unit \\ of a Tertiary Private Hospital from Recife, northeast of Brazil
}

Joana Corrêa de A. Koury ${ }^{1}$, Heloísa Ramos Lacerda², Alberto José de Barros Neto ${ }^{3}$

\section{RESUMO}

JUSTIFICATIVA E OBJETIVOS: Determinar as características clínicas, epidemiológicas e laboratoriais, bem como a mortalidade dos pacientes adultos com sepse, admitidos na UTI geral de hospital privado terciário do estado de Pernambuco.

MÉTODO: Estudo tipo coorte prospectiva e observacional, que incluiu pacientes adultos admitidos com o diagnóstico de sepse ou que a desenvolveram durante a internação na UTI do Hospital Português de Recife durante o período de seis meses. Foram colhidos dados clínicos e laboratoriais, bem como, calculados os escores APACHE II e SOFA, sendo os pacientes re-avaliados ao final da internação para definir o desfecho clínico, ou seja, para alta, óbito, ou transferência da UTI.

RESULTADOS: Foram incluídos 199 pacientes, dos quais $112(56,3 \%)$ tiveram alta da UTI. Cerca de 67,8\% da população foi composta por idosos (maiores de 65

1. Mestre em Medicina Tropical/UFPE; Hematologista da Fundação HEMOPE, Médica da UTI do Real Hospital Português.

2. Professora Doutora Adjunta do Departamento de Medicina Clínica, CCS - UFPE e da UPE, Coordenadora do Programa de Pós-Graduação em Medicina Tropical, Vice-Coordenadora da UTI do Hospital Esperança.

3. Presidente da Sociedade de Terapia Intensiva de Pernambuco (SOTIPE), Chefe da UTI do Real Hospital Português, Especialista em Terapia Intensiva pela Associação Medicina Intensiva Brasileira (AMIB).

${ }^{*}$ Recebido do Real Hospital Português, Recife, PE

Apresentado em 16 de novembro de 2005

Aceito para publicação em 23 de fevereiro de 2006

Endereço para correspondência:

Dra. Joana Corrêa de A. Koury

Real Hospital Português, Bloca Egaz Munis

Av. Agamenon Magalhães, $s / n^{\circ}$ Derby

50070-160 Recife, PE

Fone: (81) 3416-1053

E-mail: joanakoury@ig.com.br anos), a média do APACHE II foi de 18,2 $\pm 6,3$ e a média do SOFA no primeiro dia foi de $6,3 \pm 3,7$. O motivo da internação foi clínico em $85,4 \%$ dos casos e em $76,4 \%$ dos pacientes existia doença crônica associada. A sepse foi de origem pulmonar em $79,3 \%$ dos casos e $40 \%$ dos pacientes tiveram o agente etiológico isolado. Observou-se plaquetopenia (plaquetas $<100.000 /$ $\mathrm{mm}^{3}$ ) em $20,6 \%$ dos pacientes, o INR > 1,5 em 42\%, o fibrinogênio $>300 \mathrm{mg} / \mathrm{dL}$ em $81,7 \%$ e a atividade da antitrombina $<70 \%$ em $32,5 \%$ dos casos.

CONCLUSÕES: Os dados epidemiológicos de sepse nesse estudo são semelhantes àqueles recentemente descritos em populações no Sul e Sudeste do Brasil, bem como dos Estados Unidos e Europa.

Unitermos: adultos, fatores de risco, mortalidade, sepse

\section{SUMMARY}

BACKGROUND AND OBJECTIVES: Establish the clinical, epidemiological and laboratorial characteristics of septic patients in an intensive care unit (ICU) of a tertiary care private hospital from Pernambuco, in the northeast of Brazil.

METHODS: Cohort study without intervention that included adult patients admitted in the ICU with sepsis or that developed it during ICU stay, during a 6-month period. The patients were followed during all ICU stay period. Laboratory and clinical data were collected after sepsis diagnosis. ICU admission APACHE II score and the SOFA score during the three first days after inclusion in the study were collected.

RESULTS: The study included 199 patients, of whom $67.8 \%$ were older than 65 years of age. More than a half $(56.3 \%)$ survived the septic episode, mean APACHE II was $18.2 \pm 6.3$ and mean SOFA score was 6.3 \pm 3.7 . The disease related to the admission in ICU was medical in $85.4 \%$, and the majority of patients had a 
chronic associated disease. In $79.3 \%$ the lungs were the source of sepsis and in $40 \%$ of patients the etiologic agent of sepsis was isolated. Amongst laboratory tests, thrombocytopenia (platelets $<100.000 / \mathrm{mm}^{3}$ ) was observed on $20.6 \%$, fibrinogen was elevated (> $300 \mathrm{mg} / \mathrm{dL}$ ) on $81.7 \%$, and the activity of antithrombin was low $(<70 \%)$ on $32.5 \%$ of patients.

CONCLUSIONS: The epidemiology of sepsis in this study, in a private hospital of Recife, was no different from what was recently described in Southeast and South of Brazil, United States and Europe.

Key Words: adults, mortality, risk factors, sepsis

\section{INTRODUÇÃO}

Dados sobre as características dos pacientes com sepse nas Unidades de Terapia Intensiva (UTI) do Nordeste são escassos. O Brazilian Sepsis Epidemiological Study (BASES), forneceu informações epidemiológicas muito interessantes sobre a sepse nas UTI do Sul e Sudeste do país ${ }^{1}$. Silva e col. ${ }^{1}$ enfatizaram, porém, que o Brasil é um país de dimensões continentais e com uma população heterogênea sendo, por isso, necessário que cada região ou serviço de saúde saiba o real perfil epidemiológico dos pacientes com sepse sob seus cuidados, para definir prioridades de intervenção com a intenção de melhorar o atendimento a esse grupo de pacientes.

Martin e col. ${ }^{2}$, realizaram uma pesquisa nos Estados Unidos da América (EUA) onde avaliaram as mudanças epidemiológicas da sepse ocorridas em um período de 22 anos, mostrando que a prevalência dos casos de sepse aumentou $13,7 \%$ ao ano no período e que a média de idade dos pacientes acometidos aumentou de 57,4 para 60,8 anos. A sepse é a $10^{a}$ causa de morte nos EUA e a segunda nas UTI não coronarianas. Os pacientes com sepse exigem maior tempo de internação na UTI resultando num maior custo de tratamento comparado a outros pacientes. Foi estimado um gasto direto de 23.000 a 34.000 euros, por paciente com sepse, em um estudo de fármaco-economia europeu ${ }^{3}$ e Angus e col. ${ }^{4}$ observaram, nos EUA, um gasto anual total de 16,7 bilhões de dólares com pacientes que apresentavam sepse grave.

No BASES foram estudados 1383 adultos admitidos em UTI públicas e privadas do sul e sudeste do Brasil'. Destes, 884 pacientes permaneceram na UTI por mais de $24 \mathrm{~h}$ e, entre esses, $46,9 \%$ preencheram os critérios de sepse. Sendo que grande parte dos pacientes $(67,7 \%)$ já apresentava sepse na admissão da UTI.
Neste estudo, a taxa de mortalidade para os casos de sepse foi de $33,9 \%$ e sepse grave $46,9 \%$.

A lesão endotelial na sepse resulta na ativação dos fatores da coagulação seguidos pela ativação do sistema fibrinolítico que é posteriormente inibido. Este estado pró-coagulante pode se manifestar clinicamente como coagulação intravascular disseminada (CID), com larga deposição de fibrina na microvasculatura de vários órgãos, estando associado ao desenvolvimento de falência de múltiplos órgãos (FMO) e contribuindo com o prognóstico reservado destes pacientes ${ }^{5-8}$. Os baixos níveis de atividade da antitrombina (AT) e concentrações elevadas do D-dímero refletem o desenvolvimento de um estado de hipercoagulabilidade, às vezes, mais precoce que o surgimento de parâmetros clínicos usuais desta fase ${ }^{9-12}$. Yan e col. ${ }^{13}$ observaram que o D-dímero estava acima do normal em todos os pacientes com sepse grave e Wada e col. ${ }^{14}$ descreveram que os pacientes com maior número de falência de órgãos apresentavam níveis de fibrinogênio mais elevados.

A plaquetopenia é uma complicação bem conhecida na UTI e tem sido associada a vários fatores, sendo o mais importante deles, a sepse. A incidência de plaquetopenia na sepse é de $35 \%$ a $59 \%$, havendo uma correlação inversa entre a gravidade da doença e a contagem de plaquetas ${ }^{13,15,16}$.

Para conhecer melhor a realidade da sepse entre adultos na cidade do Recife foi realizado o presente estudo, cujos objetivos são descrever as características clínicoepidemiológicas e laboratoriais, bem como delinear o seu desfecho clínico durante a internação na UTI.

\section{MÉTODO}

O trabalho foi realizado na UTI geral do Real Hospital Português de Beneficência (RHP) situado na cidade de Recife-PE, Brasil. É um hospital privado de cuidados terciários com 525 leitos e uma UTI geral composta de 26 leitos. O hospital possui uma UTI cardiológica e uma UTI neurológica que não foram incluídas no estudo.

O desenho do estudo foi uma coorte prospectiva, observacional, com amostragem não-aleatória.

A pesquisa foi aprovada pela Comissão de Ética do Centro de Ciências da Saúde da Universidade Federal de Pernambuco (protocolo $N^{\circ} 187 / 2004$ ), uma vez que o RHP não possui Comitê de Ética em pesquisa. Os pacientes ou seus familiares assinaram um termo de consentimento informado.

O tamanho da amostra foi calculado no Epi-info 6.0 
com um beta $=0,2$ e um alfa $=0,05$, tendo sido estimado o tamanho mínimo da amostra para cada variável independente usada na pesquisa. Foram incluídos todos os pacientes adultos internados na UTI que apresentavam sepse no momento da internação na UTI ou desenvolvida após a admissão, no período de agosto a dezembro de 2004. Sepse foi definida como um processo infeccioso associado a dois ou mais critérios da síndrome da resposta inflamatória sistêmica: temperatura $\geq 38^{\circ} \mathrm{C}$ ou $\leq 36^{\circ} \mathrm{C}$; freqüência cardíaca $\geq 90 \mathrm{bpm}$; freqüência respiratória $\geq 20 \mathrm{mov} / \mathrm{min}$, ou $\mathrm{PaCO}_{2} \leq 32$ $\mathrm{mmHg}$, ou necessidade de ventilação mecânica; leucócitos $\geq 12.000 \mathrm{cél} / \mathrm{mm}^{3}$, ou $<4.000 \mathrm{cél} / \mathrm{mm}^{3}$, ou $10 \%$ de células imaturas; os pacientes com sepse grave além dos critérios descritos, apresentavam ao menos uma falência orgânica e choque séptico, os pacientes, além de preencherem os critérios de sepse, apresentavam pressão sistólica $\leq 90 \mathrm{mmHg}$ após reposição volêmica, com necessidade de drogas vasoativas ${ }^{19}$. Foram excluídos os pacientes que retornaram à UTI, com novo episódio de sepse, após já terem participado do estudo na internação anterior.

O foco infeccioso foi considerado como pulmonar quando havia tosse, secreção traqueal purulenta, além da presença de infiltrados intersticiais ou condensações na radiografia de tórax. A infecção urinária foi caracterizada pela presença de piúria (leucócitos $>10 / \mu \mathrm{L}$ ) no sumário de urina associada à urocultura positiva. A origem da sepse foi considerada como gastrintestinal nos pacientes com quadros de diarréia infecciosa (caracterizada pela presença de leucócitos fecais em pacientes com diarréia aguda), bem como naqueles com quadro de peritonite bacteriana espontânea ou secundária a cirurgia e diálise peritoneal. A sepse foi considerada comunitária nos pacientes com menos de $72 \mathrm{~h}$ de internação hospitalar prévia a admissão na UTI e nosocomial quando o tempo de internação era maior que $72 \mathrm{~h}$.

Todos pacientes admitidos na UTI tiveram o escore APACHE II calculado nas primeiras $24 \mathrm{~h}$ da admissão. Após o diagnóstico de sepse os pacientes eram avaliados por três dias consecutivos para coleta de dados, e re-avaliados ao final da internação na UTI para definição do desfecho clínico, alta ou óbito na UTI. A coleta de dados clínicos e laboratoriais foi realizada por um dos pesquisadores através de exame do paciente e análise da história clínica registrada em prontuário. Os testes laboratoriais foram feitos em um único laboratório.

Para o escore APACHE II foram utilizados os piores dados clínicos e laboratoriais das primeiras 24 horas da internação na UTI e as informações referentes ao SOFA foram obtidas por três dias a partir do dia em que o paciente foi incluído no estudo. Os exames de coagulação, albumina sérica e lactato foram colhidos até $48 \mathrm{~h}$ após a inclusão no estudo. Com relação aos exames microbiológicos, foram registrados os resultados relativos às primeiras culturas coletadas após o diagnóstico de sepse. Foram colhidas duas amostras de hemoculturas para pesquisa de bactérias aeróbicas, anaeróbicas e fungo. A urocultura foi considerada positiva quando apresentava mais de $10^{5} \mathrm{UFC} / \mathrm{mL}^{20}$; e a cultura de secreção traqueal (obtida por aspirado traqueal nos pacientes intubados ou traqueostomizados) quando apresentava acima de $10^{6} \mathrm{UFC} / \mathrm{mL}^{21,22}$.

A análise foi realizada com auxílio do software SPSS (Statistical Package for Social Sciences) Inc./9.0 para Windows. Foi utilizado o teste Qui-quadrado de Pearson para associação das variáveis categóricas, sendo considerado estatisticamente significativo um $p<0,05$, bem como foi calculado o odds ratio (OR) para estimativa de risco e o intervalo de confiança de 95\%. Também foi realizada uma regressão logística univariada para as variáveis contínuas como lactato, SOFA e APACHE II.

\section{RESULTADOS}

A amostra foi constituída de 199 pacientes, a maioria do sexo masculino (56,3\%), com média de idade de $68,8 \pm 16,7$ anos. A sepse foi de origem comunitária em $61,9 \%$ dos casos. Cerca de $1 \%$ dos pacientes foram classificados como sepse, $74,9 \%$ como sepse grave e $24,1 \%$ como choque séptico no momento da inclusão na pesquisa (Tabela 1), sendo que 87 (43,7\%) pacientes evoluiu para óbito e $112(56,3 \%)$ tiveram alta da UTI. A mortalidade foi de $36,3 \%$ na sepse grave e de $63,8 \%$ no choque séptico; sendo constatada uma associação entre a gravidade da sepse e o risco de morte $(p=0,002)$ (Tabela 1$)$.

Tabela 1 - Associação entre a Evolução dos Pacientes Admitidos na UTI e a Gravidade da Sepse

\begin{tabular}{l|c|c|c|c|c|c|l}
\hline \multirow{2}{*}{$\begin{array}{l}\text { Gravidade } \\
\text { da Sepse }\end{array}$} & \multicolumn{4}{|c|}{ Evolução } & \multicolumn{2}{|c|}{ Total } & \multirow{2}{*}{ p-valor } \\
\cline { 2 - 6 } & \multicolumn{2}{|c|}{ Óbito } & \multicolumn{2}{|c|}{ Alta } & \multicolumn{2}{|c|}{} \\
\cline { 2 - 6 } & $\mathrm{N}$ & $\%$ & $\mathrm{~N}$ & $\%$ & $\mathrm{~N}$ & $\%$ & \\
\hline Sepse & 00 & 0,0 & 02 & 1,8 & 02 & 1,0 & \\
Sepse grave & 53 & 63,8 & 93 & 83,0 & 146 & 74,9 & \\
Choque & 30 & 36,2 & 17 & 15,2 & 47 & 24,1 & 0,002 \\
Séptico & & & & & & & \\
Total ${ }^{\star \star}$ & 83 & 100,0 & 112 & 100,0 & 195 & 100,0 & \\
\hline
\end{tabular}

*Classificação conforme o American College of Chest Physicians/Society of Critical Care Medicine ${ }^{18}$.

**4 pacientes não foram classificados conforme a gravidade da sepse. 
A presença da sepse no momento da internação ocorreu em 145 pacientes (85,8\%). Entre os pacientes que desenvolveram sepse após a admissão, o momento da sepse foi em média de 3,4 dias, com um mínimo de um e máximo de 10 dias.

O tempo médio de permanência na UTI foi de 11,3 (1-77) dias para os pacientes que tiveram alta da UTI e de 15,7 (1-120) dias para os pacientes que foram a óbito. A maioria permaneceu menos de $72 \mathrm{~h}$ na UTI $(61,9 \%)$, destes, $37,7 \%$ foram a óbito e $62,3 \%$ tiveram alta.

Em $85,4 \%$ dos casos o motivo da internação foi clínico, em $11,1 \%$ cirúrgico, enquanto que apenas $3,5 \%$ dos pacientes foram politraumatizados. A maioria dos pacientes tinha doença crônica associada $(76,4 \%)$. Na figura 1 é possível observar as principais co-morbidades crônicas associadas ao diagnóstico de sepse.

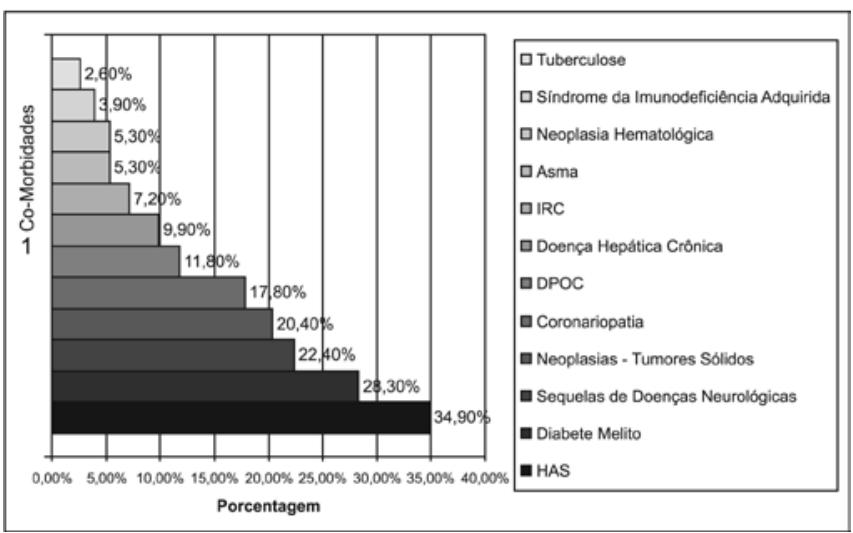

Figura 1 - Freqüência das Doenças Crônicas Diagnosticadas no Momento da Admissão na UTI.

A origem da sepse foi pulmonar em $79,3 \%$, em $14,8 \%$ dos casos foi gastrintestinal e em 13,6\% urinário. Cerca de 67 pacientes $(40,1 \%)$ tiveram o agente etiológico isolado, dos quais $25 \%$ foram Gram-positivos, 22,4\% Gram-negativos, 20,9\% de fungos e em $31,3 \%$ polimicrobianos. Na tabela 2 é possível observar quais foram os microorganismos isolados e seus respectivos percentuais.

A média do APACHE II foi de 18,2 $\pm 6,3$. Entre os pacientes com APACHE II maior que 25, cerca de $71,4 \%$ foram a óbito $(p=0,003)$. Cada elevação de 0,095 na pontuação do APACHE II estava associada a um aumento na mortalidade (OR =1,100 IC 95\% = $1,044-1,158)$. A média do SOFA no primeiro dia foi de $6,3 \pm 3,7$. Cerca de $81,1 \%$ dos casos com SOFA maior do que 12 foram a óbito $(p<0,001)$, sendo observado um aumento no risco de morte para cada elevação de 0,229 na pontuação do SOFA (OR $=1,257$ IC 95\% = 1,145-1,379) (Tabelas 3 e 4).

A falência de órgãos, definida como qualquer pontuação em algum dos parâmetros do SOFA até o $3^{\circ}$ dia da sepse, ocorreu em um a três órgãos em $54,6 \%$ dos casos. Nos $45,6 \%$ dos casos restantes observou-se falência de quatro a seis órgãos.

A contagem das plaquetas foi realizada em 155 casos, observando-se plaquetopenia (plaquetas $<100.000 /$

Tabela 2 - Agentes Etiológicos Isolados nas Primeiras Culturas após o Diagnóstico de Sepse*

\begin{tabular}{l|c|c}
\hline & Número & Porcentagem \\
\hline Agente etiológico isolado* & & \\
Acinetobacter coalceaceticus & 01 & 1,1 \\
Burkholderia cepacia & 03 & 3,3 \\
Candida albicans & 06 & 6,5 \\
Candida tropicalis & 15 & 16,3 \\
Corynebacterium specieis & 01 & 1,1 \\
Enterococcus faecalis & 06 & 6,5 \\
Enterococcus faecium & 01 & 1,1 \\
Escherichia coli & 02 & 2,2 \\
Klebisiella pneumoniae & 08 & 8,7 \\
Listeria sp & 01 & 1.1 \\
Morganella morgani & 01 & 1,1 \\
Pseudomonas aeruginosa & 14 & 15,2 \\
Staphylococcus aureus & 09 & 9,8 \\
Staphylococcus coagulase negativa & 16 & 17,4 \\
Stenotrophomonas maltophilia & 03 & 3,3 \\
Streptococcus agalactiae & 02 & 2,2 \\
Streptococcus pneumoniae & 03 & 3,3 \\
Total & 92 & 100 \\
\hline
\end{tabular}

${ }^{*}$ Referente a hemoculturas, uroculturas e culturas de secreção traqueal; não foram coletadas culturas logo após o diagnóstico de sepse em 32 pacientes; em 21 pacientes foram isolados mais de um microorganismo.

Tabela 3 - Associação dos Escores de APACHE II e SOFA com a Evolução dos Pacientes Sépticos Admitidos na UTI.

\begin{tabular}{|c|c|c|c|c|c|c|c|}
\hline \multirow[t]{3}{*}{ Variáveis } & \multicolumn{4}{|c|}{ Evolução } & \multirow[t]{3}{*}{ OR } & \multirow[t]{3}{*}{ IC (95\%) } & \multirow[t]{3}{*}{ p-valor } \\
\hline & \multicolumn{2}{|c|}{ Óbito } & \multicolumn{2}{|c|}{ Alta } & & & \\
\hline & $\mathrm{N}$ & $\%$ & $\mathrm{~N}$ & $\%$ & & & \\
\hline \multicolumn{8}{|c|}{ Escore APACHE II * } \\
\hline$\leq 25$ & 62 & 38,8 & 98 & 61,2 & 1,0 & - & \\
\hline$>25$ & 20 & 71,4 & 08 & 29,6 & 3,92 & $1,64-9,52$ & 0,003 \\
\hline Total & 82 & & 106 & & & & \\
\hline \multicolumn{8}{|l|}{ SOFA $^{\star \star}$} \\
\hline$\leq 12$ & 49 & 32,9 & 100 & 67,1 & 1,0 & - & \\
\hline$>12$ & 30 & 81,1 & 07 & 18,9 & 8,74 & $3,58-21,3$ & $<0.001$ \\
\hline Total & 79 & & 107 & & & & \\
\hline
\end{tabular}

*11 pacientes sem valor do APACHE II

**13 pacientes sem valor do SOFA 
Tabela 4 - Regressão Logística Univariada tendo como Dependente os Casos de Óbitos e como Co-Variadas o Lactato, e os Escores APACHE II e SOFA.

\begin{tabular}{l|c|c|c|c|c}
\hline Variáveis & Constante & Parâmetro & OR & IC (95\%) & p-valor \\
\hline Lactato* $^{*}$ & $-1,586$ & 0,369 & 1,446 & $1,115-1,875$ & 0,005 \\
APACHE II & $-1,996$ & 0,095 & 1,100 & $1,044-1,158$ & $<0,001$ \\
SOFA $^{* *}$ & $-1,759$ & 0,229 & 1,257 & $1,145-1,379$ & $<0,001$ \\
\hline
\end{tabular}

*Valor referente a primeira amostra coletada até 48h após o diagnóstico de sepse.

**11 pacientes sem valor do APACHE II

***13 pacientes sem valor do SOFA

Tabela 5 - Associação entre a Gravidade da Sepse e as Variáveis Laboratoriais: INR, Fibrinogênio, Antitrombina, D-dímero, Contagem de Plaquetas

\begin{tabular}{|c|c|c|c|c|c|c|c|c|c|}
\hline \multirow[t]{2}{*}{ Variável Laboratorial $^{\star}$} & \multicolumn{2}{|c|}{ Sepse } & \multicolumn{2}{|c|}{ Sepse Grave } & \multicolumn{2}{|c|}{ Choque Séptico } & \multicolumn{2}{|c|}{ Total } & \multirow[t]{2}{*}{$\mathrm{p}$-valor } \\
\hline & $\mathrm{N}$ & $\%$ & $\mathrm{~N}$ & $\%$ & $\mathrm{~N}$ & $\%$ & $\mathrm{~N}$ & $\%$ & \\
\hline $\mathrm{INR} \leq 1,5 \quad$ ** & 1 & 1,1 & 72 & 80,0 & 17 & 18,9 & 90 & 100,0 & 0,045 \\
\hline INR $>1,5$ & 0 & ,0 & 41 & 64,1 & 23 & 35,9 & 64 & 100,0 & \\
\hline Fibrinogênio $\leq 300$ mg/dL & 0 & 0 & 9 & 47,4 & 10 & 52,6 & 19 & 100,0 & 0,002 \\
\hline Fibrinogênio > 300 mg/dL & 0 & 0 & 71 & 83,5 & 14 & 16,5 & 85 & 100,0 & \\
\hline AT $>70 \% \quad * \star \star$ & 0 & 0 & 45 & 80,4 & 11 & 19,6 & 56 & 100,0 & 0,041 \\
\hline AT $\leq 70 \%$ & 0 & ,0 & 16 & 59,3 & 11 & 40,7 & 27 & 100,0 & \\
\hline D-dímero < 2000 ng/mL & 0 & ,0 & 11 & 84,6 & 2 & 15,4 & 13 & 100,0 & 0,427 \\
\hline D-dímero $\geq 2000$ ng/mL & 0 & ,0 & 12 & 70,6 & 5 & 29,4 & 17 & 100,0 & \\
\hline Plaquetas $>100.000 / \mathrm{mm}^{3}$ & 1 & 0,8 & 97 & 80,8 & 22 & 18,3 & 120 & 100,0 & 0,001 \\
\hline Plaquetas $\leq 100.000 / \mathrm{mm}^{3}$ & 0 &, 0 & 15 & 48,4 & 16 & 51,6 & 31 & 100,0 & \\
\hline
\end{tabular}

*Algumas variáveis laboratoriais apresentam um N inferior a 199, pois não foram colhidas até o período de 48h após o diagnóstico de sepse;

**International Normatizated Ratio

${ }^{\star * \star}$ Antitrombina

$\mathrm{mm}^{3}$ ) em 20,6\% dos pacientes. O INR (International Normatizated Ratio) esteve alargado $(>1,5)$ em $42 \%$, o fibrinogênio elevado ( $>300 \mathrm{mg} / \mathrm{dL}$ ) em $81,7 \%$ e a atividade da AT diminuída (<70\%) em 32,5\% dos casos. Pode-se notar uma associação entre a gravidade da sepse e a presença de INR alargado ( $p=0,045)$, redução da AT $(p=0,041)$ e plaquetopenia $(p=0,001)$ (Tabela 5).

A albumina, avaliada em 109 casos, foi menor do que 2 $\mathrm{g} / \mathrm{dL}$ em $44 \%$ da população. A creatinina estava acima de $2 \mathrm{mg} / \mathrm{dL}$ em 26,1\%. O lactato foi dosado 93 pacientes, estando elevado (maior que $2 \mathrm{mmol} / \mathrm{L}$ ) em 62,4\% dos casos. Constatou-se que o risco de morte aumentava para cada elevação do lactato em 0,369 mmol/L $(\mathrm{OR}=1,446$ IC 95\%=1,115 - 1,875) (Tabela 4).

\section{DISCUSSÃO}

A idade elevada dos pacientes admitidos no estudo (67,8\% dos pacientes com idade acima de 65 anos) foi semelhante à idade dos pacientes admitidos em UTI dos estados das regiões Sul e Sudeste do Brasil ou mesmo em países desenvolvidos como os Estados Unidos ou a França ${ }^{1,2,23}$. Em todo o mundo, os idosos adoecem mais e tem maior risco de apresentar doen- ças graves e, portanto, necessidade de internação em UTI. O presente estudo foi realizado numa UTI de Pernambuco, estado brasileiro localizado na região Nordeste, particularmente pobre e com baixos índices de desenvolvimento humano, onde a proporção de idosos (acima de 60 anos) é de apenas $8,9 \%{ }^{24}$. Este fato pode ser conseqüência de um viés de seleção, pois a pesquisa foi realizada em um hospital privado que atende uma parcela mais favorecida da população e que, mesmo em um estado do Nordeste brasileiro, certamente possui uma proporção de idosos mais elevada, semelhante à das regiões mais desenvolvidas.

A infecção respiratória foi a origem da maioria dos casos de sepse $(79,3 \%)^{1,26}$, o que pode ser um reflexo do fato de mais da metade da população estudada ter sido composta por idosos que, em geral, apresentam um risco maior de infecção respiratória ${ }^{27}$. Em pacientes com idade avançada, as presenças de co-morbidades, a diminuição da resposta imune e do mecanismo de proteção das vias aéreas, que resulta na alteração do nível de consciência e/ou reflexo de deglutição, aumentando o risco de pneumonia bacteriana.

O estudo também se assemelhou aos dados de outros centros que mostram a maioria de internações nas UTI gerais serem decorrentes de problemas clíni- 
$\cos ^{23,25}$ e a presença elevada de co-morbidades como a hipertensão arterial sistêmica, a diabete e o câncer'; estes certamente refletindo a idade elevada, ou talvez, a maior susceptibilidade da população com doenças crônicas em desenvolver complicações graves. Do mesmo modo, o estudo confirmou a universalidade da associação entre os critérios de gravidade como - APACHE II ${ }^{17}$, já bastante conhecido, mas de difícil operacionalização e o SOFA ${ }^{18}$, bem mais simples, mas menos conhecido pelos médicos, e o risco de óbito na população de pacientes com sepse ${ }^{15,17,28,29}$

Os exames mais freqüentemente alterados no início da sepse foram: a elevação do fibrinogênio $(81,7 \%)$ e do D-dímero (56,7\%), alargamento do INR (42\%) e a redução da atividade da AT (32,5\%), enquanto a plaquetopenia (20,6\%) teve menor freqüência. Essas alterações da coagulação estão associadas aos quadros mais graves de sepse, conforme observado por diversos autores ${ }^{5,30-35}$. A freqüência de plaquetopenia aqui detectada esteve abaixo da descrita por $\operatorname{Aird}^{16} \mathrm{e}$ Sthephan e Monteblack ${ }^{35}$, que relataram $35 \%$ a $55 \%$ e $23 \%$ a $41 \%$ de plaquetopenia na sepse, respectivamente, porém acima do descrito por Brun-Buisson e col. ${ }^{21}$, que observaram $4 \%$. Questiona-se se as diferenças na gravidade dos pacientes não podem ter se refletido na freqüência da plaquetopenia. $A$ dosagem do lactato sérico esteve alterada ( $>2 \mathrm{mmol} / \mathrm{L})$ em mais da metade dos pacientes $(62,4 \%)$ e foi fator de risco para o óbito, estando de acordo com o que foi descrito por Alves e col. ${ }^{36}$ e Gogos e col. ${ }^{37}$.

Observou-se elevada freqüência de pacientes com albumina muito baixa, com $44 \%$ dos pacientes com valores abaixo de $2 \mathrm{~g} / \mathrm{dL}$. Os baixos valores de albumina podem refletir a desnutrição, decorrente da idade elevada ou presença de doenças crônicas nestes pacientes, ou ser apenas um indicador de gravidade e de mau prognóstico.

A positividade das culturas na sepse é variável. A taxa de identificação dos agentes etiológicos nesta pesquisa foi semelhante ao estudo de Bilevicius e col. ${ }^{38}$, realizado no Brasil, que mostrou apenas $43 \%$ das culturas positivas; enquanto uma pesquisa francesa obteve documentação microbiológica em $71 \%$ dos pacientes $^{21}$. Le Gall e col. ${ }^{39}$ observaram que as infecções adquiridas no hospital ou na UTI eram mais documentadas microbiologicamente do que as infecções comunitárias com $71 \%, 86 \%$ e $55 \%$, respectivamente. Portanto, a baixa positividade das culturas deste estudo pode refletir um predomínio de infecções comunitárias, o uso de antibióticos prévio a coleta das cul- turas, o que reduz o crescimento bacteriano, ou técnica inadequada de coleta e cultivo das amostras. Cabe ressaltar que a principal conseqüência desta falta de identificação dos agentes é o uso de esquemas amplos e agressivos de antibióticos por tempo maior do que o necessário, pela incapacidade de proceder ao desescalonamento antimicrobiano conforme preconizado por inúmeros autores, aumentando o risco da resistência bacteriana.

Os pacientes que faleceram tiveram um tempo médio de internação na UTI maior do que os que tiveram alta da UTI, esse fato, provavelmente, não está relacionado à falência aguda de órgãos secundária ao quadro séptico inicial, mas sim à presença de co-morbidades ${ }^{1,40}$. As taxas de mortalidade nos casos de sepse grave $(36,3 \%)$ e choque séptico $(63,8 \%)$ foram semelhantes àquelas encontradas por Silva e col. ${ }^{1}$ com $47,3 \%$ e $52,2 \%$ respectivamente, bem como àquela encontrada por Angus e col. ${ }^{4}$ para pacientes com sepse grave que foram admitidos na UTI $(34,1 \%)$.

\section{CONCLUSÃO}

Os resultados dessa pesquisa refletiram o perfil epidemiológico de pacientes com sepse numa UTI privada de um hospital de grande porte, mostrando que a sepse é um problema comum em idosos, e em pacientes com co-morbidades associadas. Também chamam a atenção sobre as alterações na coagulação que, freqüentemente, são encontradas principalmente nos casos de sepse grave e choque séptico. É necessário que outros serviços de terapia intensiva do Nordeste brasileiro desenvolvam pesquisas semelhantes a fim de que possa haver comparações epidemiológicas entre as UTI da região.

\section{ABREVIATURAS}

APACHE - Acute Physiology and Chronic Health EvaIuation

AT - Antitrombina

BASES - Brazilian Sepsis Epidemiological Study

CID - Coagulação Intravascular Disseminada

FMO - Falência de Múltiplos Órgãos

IC - Intervalo de Confiança

INR - International Normatizated Ratio

OR - Odds Ratio

RHP - Real Hospital Português

SOFA - Sequential Related Organ Failure Assessment SPSS - Statistical Package for Social Sciences 


\section{REFERÊNCIAS}

01. Silva E, Pedro Mde A, Sogayar AC et al - Brazilian Sepsis Epidemiological Study (BASES study). Crit Care, 2004;8:R251-R260.

02. Martin GS, Mannino DM, Eaton S et al - The epidemiology of sepsis in the United States from 1979 through 2000. N Engl J Med, 2003;348:15461554.

03. Burchardi $\mathrm{H}, \mathrm{Schneider} \mathrm{H}$ - Economic aspects of severe sepsis: a review of intensive care unit costs, cost of illness and cost effectiveness of therapy. Pharmacoeconomics, 2004;22:793-813.

04. Angus DC, Linde-Zwirble WT, Lidicker $\mathrm{J}$ et al - Epidemiology of severe sepsis in the United States: analysis of incidence, outcome and associated costs of care. Crit Care Med, 2001;29:1303-1310.

05. Lopez-Aguirre Y, Paramo JA - Endothelial cell and hemostatic activation in relation to cytokines in patients with sepsis. Thromb Res, 1999;94:95101.

06. Levi M, Ten Cate H, van Der Poll T et al - Pathogenesis of disseminated intravascular coagulation in sepsis. JAMA, 1993;270:975-979.

07. Lorente JA, Garcia-Frade LJ, Landin L - Time course of hemostatic abnormalities in sepsis and its relation to outcome. Chest, 1993;103:15361542.

08. Mesters RM, Mannucci PM, Coppola R et al - Factor VIla and antithrombin III activity during severe sepsis and septic shock in neutropenic patients. Blood, 1996;88:881-886.

09. Friedman G, Lobo S, Rigoto O - Consenso Brasileiro de sepse. Diagnóstico. Monitorização da resposta inflamatória. www.medicinaintensiva. com.br/consenso-sepse.htm (s/d, acessado em jun 2005).

10. Shorr AF, Thomas SJ, Alkins SA et al - D-dimer correlates with proinflamatory cytokine levels and outcomes in critically ill patients. Chest, 2002;121:1262-1268.

11. Vervloet MG, Thijs LG, Hack CE - Derangements of coagulation and fibrinolysis in critically ill patients with sepsis and septic shock. Semin Thromb Hemost, 1998;24:33-44

12. Gando S, Nanzaki S, Sasaki S et al - Activation of the extrinsic coagulation pathway in patients with severe sepsis and septic shock Crit Care Med, 1998;26:2005-2009.

13. Yan SB, Helterbrand JD, Hartman DL et al - Low levels of protein $C$ are associated with poor outcome in severe sepsis. Chest, 2001;120(3):915922

14. Wada $\mathrm{H}$, Mori $\mathrm{Y}$, Okabayashi $\mathrm{K}$ et al - High plasma fibrinogen level is associated with poor clinical outcome in DIC patients. Am J Hematol, 2003;72:1-7.

15. Vincent $\mathrm{JL}$, Yagushi A, Pradier O - Platelet function in sepsis. Crit Care Med, 2002;30:(Suppl5):S313-S317.

16. Aird $\mathrm{WC}-$ The hematologic system as a marker of organ dysfunction in sepsis. Mayo Clin Proc, 2003;78:869-881.

17. Knaus WA, Draper EA, Wagner DP et al - APACHE II: a severity of disease classification system. Crit Care Med, 1985;13:818-829.

18. Vincent JL, Moreno R, Takala J et al - The SOFA (Sepsis-related Organ Failure Assessment) score to describe organ dysfunction/failure. On behalf of the Working Group on Sepsis-Related Problems of the European Society of Intensive Care Medicine. Intensive Care Med, 1996;22:707-710.

19. Bone, RC, Balk, RA, Cerra, FB et al - Definitions for sepsis and organ failure and guidelines for the use of innovative therapies in sepsis. The ACCP/SCCM Consensus Conference Committee. American College of Chest Physicians and Society of Critical Care Medicine. Chest, 1992;101:1644-1655.

20. Calandra T, Cohen J, Abraham E et al -The internacional sepsis forum consensus conference on definitions of infection in the intensive care unit. Crit Care Med, 2005,33:1538-1548.
21. Cook $D$, Mandell $L-$ Endotracheal aspiration in the diagnosis of ventilator-associated pneumonia. Chest, 2000;117:S2:S195-S197.

22. Niederman MS, Craven DE, Bonten MJ et al - Guidelines for the management of adults with hospital-acquired, ventilator-associated, and healthcare-associated pneumonia. American Thoracic Society Documents. Am J Respir Crit Care Med, 2005;171:388-416.

23. Brun-Buisson C, Doyon F, Carlet $\mathrm{J}$ et al - Incidence, risk factors, and outcome of severe sepsis and septic shock in adults: a multicenter prospective study in intensive care units. French ICU Group for Severe Sepsis. JAMA, 1995;274:968-974.

24. IBGE - Perfil dos idosos responsáveis pelos domicílios no Brasil 2000. Censo 2000. http://www.ibge.gov.br/home/estatistica/populacao/perfilidoso/perfidosos2000.pdf (acessado em 08/2005)

25. Padkin A, Goldfrad C, Brady AR et al - Epidemiology of severe sepsis occurring in the first $24 \mathrm{hrs}$ in intensive care units in England, Wales, and Northern Ireland. Crit Care Med, 2003;31:2332-2338.

26. Finfer S, Bellomo R, Lipman J et al - Adult-population incidence of severe sepsis in Australian and New Zealand intensive care units. Intensive Care Med, 2004;30:589-596.

27. Martin GS, Mannino DM, Moss M - The effect of age on the development and outcome of adult sepsis. Crit Care Med, 2006;34:15-21.

28. Garnacho-Montero J, Garcia-Garmendia JL, Barrero-Almodovar A et a - Impact of adequate empirical antibiotics therapy on the outcome of patients admitted to the intensive care unit with sepsis. Crit Care Med, 2003;31:2742-2751.

29. Brun-Buisson C, Meshaka P, Pinton P et al - EPISEPSIS: a reappraisal of the epidemiology and outcome of severe sepsis in French intensive care units. Intensive Care Med, 2004;30:580-588

30. Hesselvik JF, Blomback M, Brodin B et al - Coagulation, fibrinolysis, and kallikrein systems in sepsis: relation to outcome. Crit Care Med, 1989;17:724-733.

31. Mavrommatis AC, Theodoridis T, Economou $\mathrm{M}$ et al - Activation of the fibrinolytic system and utilization of coagulation inhibitors in sepsis: comparison with severe sepsis and septic shock. Intensive Care Med, 2001;27:1853-1859.

32. Kidokoro $\mathrm{A}$, Iba $\mathrm{T}$, Fukunaga $\mathrm{M}$ et al - Alterations in coagulation and fibrinolysis during sepsis. Shock, 1996;5:223-228.

33. Leithauser B, Matthias FR, Nicolai U et al - Hemostatic abnormalities and severity of illness in patient at onset of clinically defined sepsis. Possible indication of the degree of endothelial cell activation? Intensive Care Med, 1996;22:631-636.

34. Pettila V, Pentti J, Pettila $\mathrm{M}$ et al - Predictive value of antithrombin III and serum C-reactive protein concentration in critically ill patients with suspected sepsis. Crit Care Med, 2002;30:271-275.

35. Sthephan F, Monteblanc J, Cheffi A et al - Thrombocytopenia in critically ill surgical patients: a case-control study evaluating attributable mortality and transfusion requirements. Crit Care, 1999;3:151-158.

36. Alves $\mathrm{F}$, Sant'Anna UL, Oliveira $\mathrm{E}$ et al - $\mathrm{O}$ valor prognóstico do curso hemodinâmico inicial de pacientes com falência circulatória. Rev Bras Ter Intens, 1998;10:68-75.

37. Gogos CA, Lekkou A, Papageorgiou O - Clinical prognostic markers in patients with severe sepsis: a prospective analysis of 139 consecutive cases. J Infect, 2003;47:300-306.

38. Bilevicius E, Dragosavac D, Dragosavac $S$ et al - Multiple organ failure in septic patient. Braz J Infect Dis, 2001;5:103-110.

39. Le Gall JR, Alberti C, Brun-Buisson C - Epidemiology of infection and sepsis in intensive care unit patients. Bull Acad Natl Med, 2004;188:11151125.

40. Alberti C, Brun-Buisson C, Goodman SV et al - Influence of systemic inflammatory response syndrome and sepsis on outcome of critically ill infected patients. Am. J. Respir Crit Care Med, 2003;68:77-84. 